\title{
Hospitalización de 780 episodios de infección en 10 servicios de urgencias españoles. ¿Ingreso convencional o unidad de corta estancia?
}

\author{
Hospitalization of 780 episodes of infection in 10 Spanish emergency \\ departments. Admission to conventional wards or short stay units?
}

\author{
F. Llopis Roca ${ }^{1}$, A. Juan Pastor ${ }^{2}$, C. Ferré Losa ${ }^{3}, J^{\text {. González del Castillo }}{ }^{4}$, M. Ruiz Grinspan ${ }^{5}$, \\ M. Martínez Ortíz de Zárate ${ }^{6}$ (en representación del grupo INFURG-SEMES)
}

\section{RESUMEN}

Fundamento. Determinar las características clínicas de los enfermos con infección que ingresan desde urgencias y comparar las que lo hacen en una unidad de corta estancia (UCE) con las unidades de hospitalización convencional (UHC).

Material y métodos. Estudio descriptivo multicéntrico con análisis transversal en 10 servicios de urgencias con UCE de pacientes con infección que ingresan. Se analizó edad, género, comorbilidad, factores de riesgo para patógenos multirresistentes, tipo de infección, criterios de sepsis, microbiología y antibioticoterapia.

Resultados. Ingresaron 780 enfermos; edad media 70,43 años; $31 \%$ con cardiopatía, $29 \%$ con EPOC, $26 \%$ con diabetes mellitus, $15 \%$ con antibioticoterapia previa y neoplasia sólida. El 54\% eran infecciones respiratorias, $22 \%$ urinarias y $8 \%$ intraabdominales. El $13 \%$ presentó síndrome séptico y los antibióticos más prescritos fueron betalactámicos (64\%) y fluorquinolonas (29\%). Al comparar los episodios que ingresaron en UCE (183) respecto UHC (597), estos últimos tenían más comorbilidad ( $86 \%$ vs. $78 \%$ ) y factores de riesgo de patógenos multirresistentes ( $42 \%$ vs. $25 \%$ ) y se practicaban más cultivos $(80 \%$ vs. $64 \%)(\mathrm{p}<0,05)$.

Conclusiones. Las UCE son una excelente alternativa a las UHC para enfermos con infección prevalente y con menor comorbilidad y factores de riesgo de multirresistencia.

Palabras clave. Infección. Urgencias. Unidad de corta estancia.

\begin{abstract}
Background. To study the clinical characteristics of patients with infection attending the emergency department (ED) and compare those admitted to a short stay unit (SSU) with those admitted to a conventional hospital ward (CHW).
\end{abstract}

Methods. A descriptive multicenter cross-sectional analysis of infected patients requiring admission from 10 ED with SSU. Data were collected for age, gender, comorbidities, risk factors for multiresistant pathogens, type of infection, sepsis criteria, microbiology and antibiotic treatment.

Results. We documented 780 admitted patients, mean age 70.43 years, $31 \%$ with heart disease, $29 \%$ COPD, $26 \%$ diabetes mellitus, $15 \%$ prior antibiotic therapy and solid neoplasm. Fifty-four percent were respiratory infections, $22 \%$ urinary infections and $8 \%$ intra-abdominal infections. Thirteen percent had septic syndrome and beta-lactam (64\%) and fluoroquinolones (29\%) were the most prescribed antibiotics. When comparing patients admitted to SSU (183) with those admitted to CHW (597), in the latter group there were more comorbidities (86\% vs. $78 \%$ ), more risk factors for multidrug-resistant pathogens ( $42 \%$ vs. $25 \%$ ) and cultures from different sources were more frequently undertaken ( $80 \%$ vs. $64 \%)(\mathrm{p}<0.05)$.

Conclusions. According to our results, SSU may be an excellent alternative to $\mathrm{CHW}$ for patients with prevalent infection and less comorbidity and fewer risk factors for multidrug resistance.

Keywords. Infection. Emergency. Short stay unit.
1. Hospital Universitari de Bellvitge. L'Hospitalet de Llobregat. Barcelona.

2. Institut Català de la Salut. Coordinador del grupo de trabajo URG-UCE-SEMES.

3. Hospital Universitari de Bellvitge. L'Hospitalet de Llobregat. Barcelona.

4. Hospital Clínico San Carlos. Madrid. Coordinador del grupo de trabajo INFURG-SEMES.

5. Hospital de Henares. Coslada. Madrid.

6. Hospital Universitario de Basurto. Bilbao.

\section{Correspondencia:}

Ferran Llopis Roca

Servicio de Urgencias

Hospital Universitari de Bellvitge

Feixa Llarga s/n

08907 L'Hospitalet de Llobregat. Barcelona

E-mail: fllopis@bellvitgehospital.cat

Recepción: 24 de septiembre de 2014

Aceptación provisional: 4 de noviembre de 2014

Aceptación definitiva: 17 de diciembre de 2014 


\section{INTRODUCCIÓN}

De acuerdo con los estudios publicados en las tres últimas décadas, las infecciones en España representan entre un 5 y $17 \%$ de las visitas a los servicios de urgencias hospitalarias (SUH) ${ }^{1,2}$. El grupo de trabajo de Enfermedades Infecciosas de la Sociedad Española de Medicina de Urgencias y Emergencias (INFURG-SEMES) ha publicado recientemente un estudio sobre la epidemiología de las infecciones en los SUH y su evolución en la última década $^{3}$. En éste se objetiva una prevalencia global del $14,3 \%$, lo que supone un aumento de 4 puntos porcentuales respecto al registro realizado por el mismo grupo 13 años antes, con una prevalencia del $10,4 \%{ }^{4}$.

Por otro lado, recientemente se han publicado los resultados del estudio RE$\mathrm{GICE}^{5,6}$, en el que se aportan datos sobre estructura, distribución de profesionales y resultados de actividad y de gestión clínica de las unidades de corta estancia (UCE). En este trabajo se pone de manifiesto que del total de 45.140 altas reportadas en las UCE españolas durante un año, 3 GRD asociados con patología infecciosa se sitúan entre los primeros 10 diagnósticos al alta: GRD 321 (infecciones del riñón y tracto urinario, edad > 18 años sin comorbilidad), GRD 541 (neumonía simple y otros trastornos respiratorios excepto bronquitis y asma con comorbilidad) y GRD 320 (infecciones del riñón y tracto urinario). En este sentido, existe experiencia en patología infecciosa ingresada en UCE, como por ejemplo la neumonía ${ }^{7,8}$, con muy buenos resultados atendiendo al perfil de seguridad (mortalidad y reingreso) y satisfacción de los pacientes.

El presente trabajo tiene como objetivo, a partir del estudio epidemiológico de INFURG-SEMES, determinar cuáles son las características clínicas de los enfermos con infección atendidos en urgencias que requieren ingreso y comparar las infecciones de pacientes que ingresan en una UCE respecto a las de los que lo hacen en una unidad de hospitalización convencional (UHC).

\section{MATERIAL Y MÉTODOS}

El presente trabajo es un estudio descriptivo, multicéntrico, prospectivo y observacional, realizado en 49 SUH españoles, de los procesos infecciosos atendidos los días 10 (de las $0 \mathrm{~h}$ a las $12 \mathrm{~h}$ ) y 20 (de las $12 \mathrm{~h}$ a las 24h) de cada mes durante 1 año (de octubre de 2010 a septiembre de 2011). A efectos del presente estudio se comparan los pacientes que ingresaron en una UCE respecto los que lo hicieron en una UHC.

Se seleccionan 10 hospitales que disponen de UCE, que forman el grupo "Estudio INFURG-SEMES": Hospital de Sant Pau (Josep Maria Guardiola, Verónica Díez), Hospital Vall d'Hebron (Teresa Soriano), Hospital de Bellvitge (Javier Jacob), Hospital Moisès Broggi (Pablo Marchena), Hospital Mútua de Terrassa (Ana Álvarez), Hospital Verge de la Cinta de Tortosa (Manel Chánovas), Hospital Clínico San Carlos (Francisco Javier Martín, Francisco Javier Candel, Eric Jorge García-Lamberechts), Hospital Severo Ochoa (Beatriz Valle), Hospital de Basurto (Juan Larruskain), Hospital del Bierzo (María José Antequera).

Se recogieron las siguiente variables: edad, género, comorbilidad, factores de riesgo de patógenos multirresistentes, ingreso hospitalario los 3 meses previos o institucionalización, criterios clínicos de sepsis, pruebas microbiológicas solicitadas y diagnóstico clínico y antibiótico prescrito en urgencias.

Para describir las variables cualitativas se utilizó la frecuencia absoluta y relativa de cada uno de los valores de las variables, y para las variables cuantitativas la media y la desviación estándar. Las comparaciones se hicieron mediante razones de prevalencia (RP) o diferencias de medias respectivamente, con su intervalo de confianza del $95 \%$ (IC $95 \%$ ). Un valor de $\mathrm{p}<0,05$ o cuando el IC 95\% de la RP excluyó el valor 1 o el 0 para el IC 95\% de la diferencia se consideró estadísticamente significativo. Para el análisis comparativo se utilizó el programa SPSS 21.0 aplicando tablas de contingencia, con la prueba chi cuadrado para las variables cualitativas y la $t$ de Student para comparar medias en las variables cuantitativas, con 
diferencias estadísticamente significativas cuando el valor de la $p$ era inferior a 0,05 .

El estudio epidemiológico fue aprobado por el Comité de Ensayos Clínicos e Investigación del Hospital Universitario de Basurto.

\section{RESULTADOS}

Durante el periodo de estudio se describieron 11.399 infecciones entre las 79.654 asistencias realizadas en urgencias lo que supone una prevalencia del $14,3 \%$ y 2.923 (26\%) de estos episodios correspondieron a 10 hospitales que disponen de UCE, todas ellas con una dependencia funcional del servicio de urgencias. De estos 2.923 episodios, requirieron ingreso 780 (27\%) pacientes, ya fuese en una UHC (597 casos) o en una UCE (183 casos) (Fig. 1).

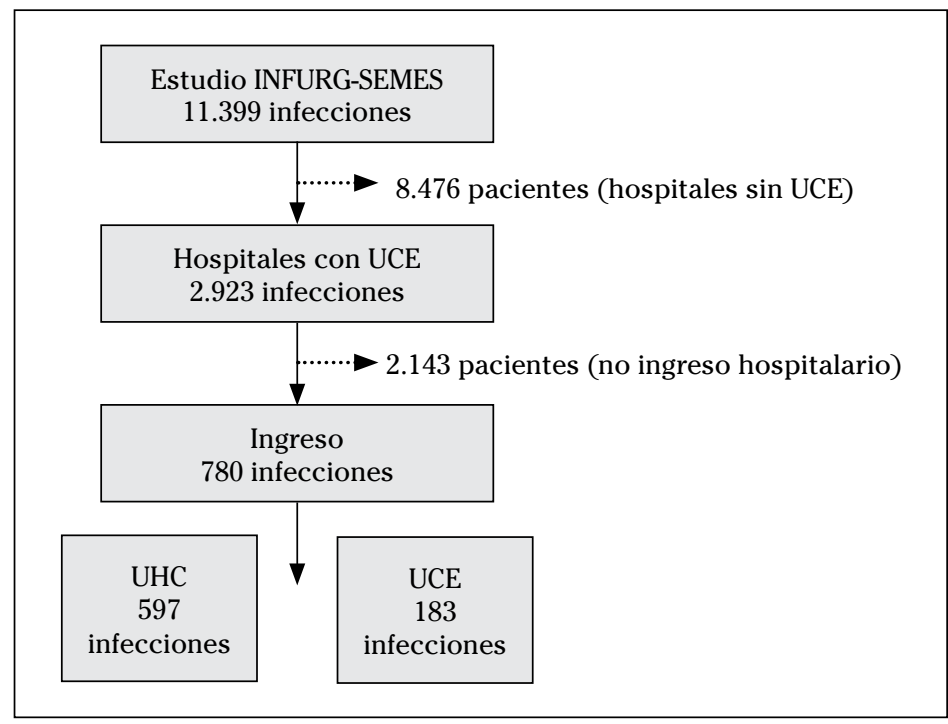

UCE: Unidad de corta estancia

UHC: Unidad de hospitalización convencional

Figura 1. Flujograma de los pacientes incluidos en el estudio.

Al comparar los episodios de infección que ingresaron en las UCE con los que ingresaron en las UHC, se observa de forma estadísticamente significativa que en el segundo grupo los pacientes presentaban mayor comorbilidad (86\% frente a $78 \%$, $\mathrm{p}=0,011)$, más factores de riesgo para patógenos multirresistentes (42\% frente a $25 \%$, $\mathrm{p}<0,001)$ y se les practicaron más cultivos ( $80 \%$ frente a $64 \%, \mathrm{p}<0,001$ ) (Tabla 2). Si analizamos el modelo de infección más frecuente, la exacerbación infecciosa de la
EPOC, que supone el $16 \%$ del total de las infecciones (127 casos), se mantienen las diferencias en la presencia de mayor comorbilidad ( $86 \%$ frente a $64 \%, p=0,010)$ y la práctica de más cultivos (66\% frente a $40 \%$, $\mathrm{p}=0,008$ ) en el grupo que ingresa en las UHC respecto las UCE. Sin embargo, a pesar de que se mantienen los porcentajes en los factores de riesgo para patógenos multirresistentes (64\% frente a $48 \%$ ) se pierde la significación estadística $(\mathrm{p}=0,125)$. 
La tabla 1 refleja las principales carac-

terísticas clínicas de estos 780 episodios.

Tabla 1. Características clínicas de los 780 episodios de infección que requieren ingreso en UHC o UCE.

\begin{tabular}{|c|c|}
\hline Edad media (años \pm DE) & $70,43 \pm 17,98$ \\
\hline Género (hombre, \%) & $420(53,8)$ \\
\hline \multicolumn{2}{|l|}{ Condiciones de comorbilidad n (\%) } \\
\hline Cardiopatía & $245(31,4)$ \\
\hline EPOC & $228(29,2)$ \\
\hline Diabetes mellitus & $207(26,5)$ \\
\hline Neoplasia sólida & $115(14,7)$ \\
\hline Insuficiencia renal crónica & $87(11,2)$ \\
\hline Hepatopatía & $34(4,4)$ \\
\hline Neoplasia hematológica & $26(3,3)$ \\
\hline Infección por el VIH & $11(1,4)$ \\
\hline Otros antecedentes no especificados & $284(36,4)$ \\
\hline \multicolumn{2}{|l|}{ Factores de riesgo de patógenos multirresistentes $\mathrm{n}(\%)$} \\
\hline Antibiótico el mes previo & $120(15,4)$ \\
\hline Ingreso previo & $94(12,1)$ \\
\hline Tratamiento inmunosupresor o quimioterapia & $85(10,9)$ \\
\hline Institucionalización / manipulación previa & $76(9,7)$ \\
\hline Tratamiento anticoagulante & $70(9)$ \\
\hline Sonda uretral & $32(4,1)$ \\
\hline Catéter central & $9(1,2)$ \\
\hline Consumo de drogas por vía parenteral & $3(0,4)$ \\
\hline Presentación como síndrome séptico n (\%) & $99(12,7)$ \\
\hline Sepsis & $71(9,1)$ \\
\hline Sepsis grave & $21(2,7)$ \\
\hline Shock séptico & $7(0,9)$ \\
\hline \multicolumn{2}{|l|}{ Tipo de infección $\mathrm{n}(\%)$} \\
\hline Respiratorias & $418(53,6)$ \\
\hline Urinarias & $173(22,2)$ \\
\hline Intraabdominales & $65(8,3)$ \\
\hline Piel y partes blandas & $46(5,9)$ \\
\hline Otras & $78(10)$ \\
\hline \multicolumn{2}{|l|}{ Estudio microbiológico realizado $\mathrm{n}(\%)$} \\
\hline Hemocultivos & $356(45,6)$ \\
\hline Urocultivo & $244(31,3)$ \\
\hline Antígenos en orina & $139(17,8)$ \\
\hline Cultivo de muestras respiratorias & $119(15,3)$ \\
\hline Otros cultivos & $99(12,7)$ \\
\hline Sin estudio microbiológico realizado & $187(24)$ \\
\hline \multicolumn{2}{|l|}{ Tratamiento antibiótico prescrito $\mathrm{n}(\%)$} \\
\hline Betalactámicos & $500(64,1)$ \\
\hline Quinolonas & $225(28,8)$ \\
\hline Anaerobicidas (metronidazol, clindamicina) & $38(4,9)$ \\
\hline Carbapenémico & $58(7,4)$ \\
\hline Macrólido & $13(1,7)$ \\
\hline Aminoglicósidos & $5(0,6)$ \\
\hline Glucopéptido & $5(0,6)$ \\
\hline Sin tratamiento antibiótico prescrito & $17(2,2)$ \\
\hline
\end{tabular}


Tabla 2. Análisis comparativo de las infecciones que ingresan en las UCE y las que ingresan en las UHC

\begin{tabular}{|c|c|c|c|}
\hline Características & UCE (N=183) & UHC (N=597) & $\mathbf{P}$ \\
\hline Edad media $($ años \pm DE) & $69,34 \pm 19,6$ & $70,76 \pm 17,45$ & 0,382 \\
\hline Género (hombres, \%) & 49,72 & 55,11 & 0,205 \\
\hline $\begin{array}{l}\text { Comorbilidad (\%) } \\
\text { Cardiopatía } \\
\text { EPOC } \\
\text { Diabetes } \\
\text { Neoplasia sólida } \\
\text { Insuficiencia renal crónica } \\
\text { Hepatopatía } \\
\text { Neoplasia hematológica }\end{array}$ & $\begin{array}{c}77,6 \\
32,24 \\
30,6 \\
19,13 \\
8,74 \\
6,01 \\
1,64 \\
1,1\end{array}$ & $\begin{array}{c}85,93 \\
31,16 \\
28,81 \\
28,81 \\
16,58 \\
12,73 \\
5,19 \\
4,02\end{array}$ & $\begin{array}{l}0,011 \\
0,785 \\
0,643 \\
0,010 \\
0.009 \\
0,011 \\
0,039 \\
0,059\end{array}$ \\
\hline $\begin{array}{l}\text { Factores de riesgo de patógenos multiresistentes (\%) } \\
\text { Tratamiento inmunosupresor o quimioterapia } \\
\text { Sonda uretral } \\
\text { Ingreso previo } \\
\text { Institucionalización/instrumentalización } \\
\text { Antibioticoterapia el último mes }\end{array}$ & $\begin{array}{c}25,14 \\
3,83 \\
3,28 \\
7,1 \\
6,01 \\
9,29\end{array}$ & $\begin{array}{c}41,71 \\
13,07 \\
4,36 \\
13,57 \\
10,89 \\
17,25\end{array}$ & $\begin{array}{c}<0,001 \\
<0,001 \\
0,671 \\
0,019 \\
0,063 \\
0,010\end{array}$ \\
\hline Diagnósticos (\%) & & & \\
\hline $\begin{array}{l}\text { Pielonefritis } \\
\text { Vías bajas } \\
\text { Infección en portador de sonda vesical }\end{array}$ & $\begin{array}{c}13,11 \\
9,29 \\
1,09\end{array}$ & $\begin{array}{c}6,53 \\
10,22 \\
2,68\end{array}$ & $\begin{array}{l}0,008 \\
0,780 \\
0,270\end{array}$ \\
\hline $\begin{array}{l}\text { Infección respiratoria } \\
\text { Bronquitis aguda } \\
\text { EPOC agudizado } \\
\text { Neumonía } \\
\text { Bronquiectasias infectadas } \\
\text { Absceso pulmonar }\end{array}$ & $\begin{array}{c}22,95 \\
22,95 \\
18,03 \\
0 \\
0\end{array}$ & $\begin{array}{c}10,72 \\
14,24 \\
24,62 \\
0,84 \\
0,17\end{array}$ & $\begin{array}{c}<0,001 \\
0,008 \\
0,071 \\
0,596 \\
1\end{array}$ \\
\hline $\begin{array}{l}\text { Infección intraabdominal } \\
\text { Infección hepatobiliar } \\
\text { Diverticulitis } \\
\text { Peritonitis primaria }\end{array}$ & $\begin{array}{c}0 \\
3,82 \\
0\end{array}$ & $\begin{array}{l}6,37 \\
1,84 \\
1,72\end{array}$ & $\begin{array}{c}<0,001 \\
0,155 \\
0,209\end{array}$ \\
\hline $\begin{array}{l}\text { Infección de piel y partes blandas } \\
\text { No necrosante } \\
\text { Necrosante } \\
\text { Pie diabético } \\
\text { Úlceras por presión }\end{array}$ & $\begin{array}{c}4,92 \\
0,55 \\
0,55 \\
0\end{array}$ & $\begin{array}{c}4,35 \\
0,33 \\
0,5 \\
0,67\end{array}$ & $\begin{array}{l}0,688 \\
0,552 \\
1 \\
0,578\end{array}$ \\
\hline $\begin{array}{l}\text { Síndrome séptico (\%) } \\
\text { Sepsis } \\
\text { Sepsis grave } \\
\text { Shock séptico }\end{array}$ & $\begin{array}{c}14,37 \\
12,64 \\
1,15 \\
0,57\end{array}$ & $\begin{array}{l}13,43 \\
8,89 \\
3,45 \\
1,09\end{array}$ & $\begin{array}{l}0,800 \\
0,145 \\
0,190 \\
1\end{array}$ \\
\hline $\begin{array}{l}\text { Realización de estudio microbiológico (\%) } \\
\text { Hemocultivos } \\
\text { Urocultivo } \\
\text { Cultivo de muestras respiratorias } \\
\text { Antígenos en orina } \\
\text { Otros cultivos } \\
\text { No realizado }\end{array}$ & $\begin{array}{c}28,41 \\
28,41 \\
9,84 \\
21,31 \\
8,2 \\
35,52\end{array}$ & $\begin{array}{l}50,92 \\
32,16 \\
16,92 \\
16,75 \\
14,07 \\
20,44\end{array}$ & $\begin{array}{c}<0,001 \\
0,363 \\
0,019 \\
0,185 \\
0,042 \\
<0,001\end{array}$ \\
\hline $\begin{array}{l}\text { Tratamiento antibiótico prescrito (\%) } \\
\text { Quinolonas (moxi / levo / ciprofloxacino) } \\
\text { Amoxicilina-clavulánico } \\
\text { Ceftriaxona / cefotaxima } \\
\text { Piperacilina-tazobactam } \\
\text { Ertapenem } \\
\text { Imipenem / meropenem } \\
\text { Glucopéptidos } \\
\text { Metronidazol } \\
\text { Clindamicina } \\
\text { Aminoglicósido } \\
\text { Sin tratamiento antibiótico }\end{array}$ & $\begin{array}{c}37,16 \\
31,69 \\
24,04 \\
0,55 \\
4,37 \\
0 \\
0 \\
0 \\
2,19 \\
3,28 \\
2,86\end{array}$ & $\begin{array}{c}26,3 \\
31,16 \\
18,76 \\
8,54 \\
2,51 \\
5,86 \\
0,84 \\
4,02 \\
1,84 \\
2,69 \\
2,18\end{array}$ & $\begin{array}{c}0,005 \\
0,927 \\
0,139 \\
<\mathbf{0 , 0 0 1} \\
0,212 \\
<\mathbf{0 , 0 0 1} \\
0,596 \\
0,002 \\
0,761 \\
0,617 \\
1\end{array}$ \\
\hline
\end{tabular}




\section{DISCUSIÓN}

Según los resultados de nuestro estudio, las UCE son una unidad de destino para pacientes con patología infecciosa procedentes de los SUH que requieren hospitalización. En nuestro trabajo hasta un $6,3 \%$ de las infecciones atendidas en urgencias ingresaron en una UCE, lo que supone un $20 \%$ del total de infecciones que requieren hospitalización.

Las UCE atienden pacientes con diversas patologías agudas y crónicas agudizadas de baja y mediana complejidad en los que habitualmente es previsible el alta a los 3-4 días ${ }^{9}$. Estas unidades suponen una alternativa a la hospitalización convencional que contribuyen a paliar la saturación de los $\mathrm{SUH}^{10}$ y que se han mostrado como un recurso seguro y eficaz en el tratamiento de patología infecciosa como la neumonía ${ }^{7,8}$, la infección urinaria ${ }^{11}$ o la de piel y partes blandas ${ }^{12}$, principales diagnósticos de ingreso en nuestro estudio. En este sentido permiten monitorizar la evolución clínica y la respuesta al tratamiento antibiótico parenteral las primeras $24-48$ horas a la espera del resultado, en su caso, de los estudios microbiológicos $\mathrm{y}$, dado que los circuitos de alta e ingreso son más ágiles que en las UHC, puede acortar estancias hospitalarias $^{7,13}$.

En cuanto a la decisión sobre el ingreso en una UHC o una UCE, se puede tomar en base a recomendaciones de guías clínicas, a protocolos establecidos o según el criterio del facultativo responsable que decide el ingreso desde el SUH. En nuestra serie, hasta un $64 \%$ de la infección que ingresa en una UCE (51\% en las UHC) corresponde a infección respiratoria en forma de bronquitis aguda, exacerbación infecciosa de la EPOC o neumonía; le siguen las infecciones urinarias $(23,5 \%)$ y de piel y partes blandas (6\%), con porcentajes equiparables a las de las UHC. En cambio, la infección intraabdominal, de manejo más complejo y con mayor probabilidad de complicaciones o de requerir tratamiento quirúrgico, como es el caso de la infección hepatobiliar, se ingresa preferentemente en las UHC. Es importante señalar que el síndrome séptico está pre- sente globalmente en un $13 \%$ de casos que requieren ingreso y sin diferencias en los enfermos que ingresan en UCE o UHC. Sin embargo, en las UHC son más frecuentes la sepsis grave y el shock séptico y es mayor el número de hemocultivos u otros cultivos en general. Por tanto, vistos los resultados, la decisión de ingreso en una UCE parece adecuada, teniendo en cuenta que lo hacen pacientes con patologías en los que estas unidades han demostrado buenos resultados en experiencias anteriores ${ }^{13,14}$, a la vez que los pacientes que ingresan presentan menor gravedad y comorbilidad.

En cuanto al tratamiento antibiótico los más frecuentemente indicados fueron los betalactámicos y las quinolonas independientemente del destino, aunque las quinolonas fueron significativamente más prescritas en las UCE, probablemente por la alta prevalencia de infección respiratoria en estas unidades. Como era previsible, antibióticos de amplio espectro y exclusivamente de administración parenteral como carbapenémicos o piperacilina-tazobactam, se utilizaron mayoritariamente en las UHC, salvo en el caso del ertapenem indicado, entre otras, en las infecciones producidas por bacterias productoras de betalactamasas de espectro extendido que facilitan la opción de completar el tratamiento con los equipos de atención domiciliaria ${ }^{15}$.

A tenor de los resultados, el perfil del paciente con infección que se beneficiaría de una UCE sería aquel enfermo con infección prevalente, especialmente la infección respiratoria y la infección urinaria, con menor comorbilidad (aunque la EPOC y la IC son igual de frecuentes en los pacientes ingresados en las UCE y las UHC), sin factores de riesgo de patógenos multirresistentes y que se presenta con sepsis no grave en urgencias. Por el contrario, los pacientes con infecciones por microorganismos multirresistentes, sospecha de infección intraabdominal o especial comorbilidad, que suelen requerir estancias superiores, no serían pacientes idóneos para su ingreso en las UCE.

Aunque nuestro estudio supone una aproximación a las infecciones atendidas en los SUH en España que ingresan en las 
UCE, tiene una serie de limitaciones. En primer lugar el propio diseño del estudio, con ciertas carencias en lo que se refiere al diagnóstico y tratamiento, como la ausencia de resultados microbiológicos o de sensibilidad antibiótica. Por otra parte, existe una falta de seguimiento de los pacientes que ingresan, que no permite conocer cuáles han sido trasladados de una UCE a una UHC por mala evolución clínica o por necesidad de prolongar la estancia hospitalaria.

A pesar de ello, la información recogida en el presente estudio pretende ser de utilidad para conocer cuáles son las características del paciente con enfermedad infecciosa que ingresa en una UCE. La infección es una patología muy prevalente en los $\mathrm{SUH}$, que ha aumentado de manera significativa en la última década y condiciona un número importante de ingresos hospitalarios, y en este sentido las UCE son una excelente alternativa a la hospitalización convencional para aquellos enfermos con menos comorbilidad y sin factores de riesgo de multirresistencia y que se benefician de un control evolutivo las primeras $24-48$ horas de tratamiento.

\section{ADENDA}

Hospitales que participaron en el estudio: Hospital de Sant Pau (Josep Maria Guardiola, Verónica Díez), Hospital Vall d'Hebron (Teresa Soriano), Hospital de Bellvitge (Javier Jacob), Hospital Moisès Broggi (Pablo Marchena), Hospital Mútua de Terrassa (Ana Álvarez), Hospital Verge de la Cinta de Tortosa (Manel Chánovas), Hospital Clínico San Carlos (Francisco Javier Martín, Francisco Javier Candel, Eric Jorge García-Lamberechts), Hospital Severo Ochoa (Beatriz Valle), Hospital de Basurto (Juan Larruskain), Hospital del Bierzo (María José Antequera).

\section{BIBLIOGRAFÍA}

1. Lloret Carbó J, Puig Puyol X, Muñoz Casadevall J. Urgencias médicas. Análisis de 18.240 pacientes atendidos en el servicio de urgencias de un hospital general en el periodo de un año. Med Clin (Barc) 1984; 83:135-141.
2. Olivan Ballabriga AS, Agudo Pascual O, Agorreta Ruiz J, Pinillos Echevarría MA, Etxebarría Lus M, Moros Borgoñón MA et al. La patología infecciosa en el servicio de urgencias de un hospital terciario. Emergencias 1998; 10: S 22-25.

3. Martínez Ortíz de Zárate M, GonzÁlez del Castillo J, Julián Jiménez A, Piñera Salmerón P, Llopis Roca F, Guardiola Tey JM et al. Estudio INFURG-SEMES: Epidemiología de las Infecciones en los Servicios de Urgencias Hospitalarios y evolución durante la última década. Emergencias 2013; 25: 368-378.

4. GRUPo Para El Estudio dE la INFECCIÓN EN URGENCIAS. Estudio epidemiológico de las infecciones en el Área de Urgencias. Emergencias 2000; 12: 80-90.

5. Llopis Roca F, Juan Pastor A, Ferré Losa C, MarTín Sánchez FJ, Llorens Soriano P, Sempere MonTES G et al. Proyecto REGICE: registro de las unidades de corta estancia en España. Localización, aspectos estructurales y dotación de profesionales (REGICE 1). Emergencias 2014; 26: 57-60.

6. Llopis Roca F, Ferré Losa C, Juan Pastor A, Martín SÁnchez FJ, Sempere Montes G, Llorens SoriANo P et al, en representación del Grupo de colaboradores del proyecto REGICE. Proyecto REGICE. Gestión Clínica de las Unidades de Corta Estancia en España (REGICE 2). Emergencias 2014; 26: 359-362.

7. Juan A, Jacob J, Llopis F, Gómez-Vaquero C, Ferré C, PÉrEz-MAs JR et al. Análisis de la seguridad y la eficacia de una unidad de corta estancia en el tratamiento de la neumonía adquirida en la comunidad. Emergencias 2011; 23: 175-182.

8. Juan Pastor A, Ferré Losa C, Llopis Roca F, Jacob Rodríguez J, Bardés Robles I, SAlazar Soler A. La unidad de corta estancia como alternativa a la hospitalización convencional en el tratamiento de la neumonía adquirida en la comunidad en la población mayor de 75 años. Rev Esp Geriatr Gerontol 2011; 46: 213-216.

9. Alonso G, Escudero JM. La unidad de corta estancia de urgencias y la hospitalización a domicilio como alternativas a la hospitalización convencional. An Sist Sanit Navar 2010; 33 (Supl 1): 97-106.

10. JuAn PAStor A. Las Unidades de Corta Estancia médicas. Rev Calid Asist 2013; 28: 197-198.

11. Gordo Remartínez S, Nuevo González JA, Cano Ballesteros JC, Sevillano Fernández JA, Granda Martín MJ, Audibert Mena L. Características de la pielonefritis aguda en una unidad de corta estancia. Rev Clin Esp 2009; 209: 382-387.

12. Juan A, Salazar A, Álvarez A, PÉrez JR, Garcia L, Corbella X. Effectiveness and safety of an emergency department short-stay unit as an 
alternative to standard inpatient hospitalisation. Emerg Med J 2006; 23: 833-837.

13. Sempere Montes G, Morales Suárez Varela M, Garijo Gómez E, Illa Gómez MD, Palau Muñoz P. Impacto de una unidad de corta estancia en un hospital de tercer nivel. Rev Clin Esp 2010; 210: 279-283.

14. González Armengol JJ, Fernández Alonso C, Martín Sánchez FJ, González del Castillo J, LóPEZ FARré A, ELVIRA C et al. Actividad de una unidad de corta estancia en urgencias en un hospital terciario: cuatro años de experiencia. Emergencias 2009; 21: 87-94.

15. Mujal Martínez A, Solá Aznar J, Hernández Ávila M, AragüÁs Flores C, Machado Sicilia ML, OrisTRELl SALVÁ J. Eficacia y seguridad del tratamiento antibiótico domiciliario endovenoso en pacientes con patología infecciosa procedentes del servicio de urgencias. Emergencias 2013; 25: 31-36. 